\title{
UNIFORM ORDER-CONVERGENCE FOR COMPLETE LATTICES
}

\author{
GABRIELE H. GRECO
}

\begin{abstract}
We introduce a purely lattice-theoretical definition of uniform orderconvergence of a net of functions with values in a complete lattice. We will show that for completely distributive lattices the uniform order-convergence is induced by a uniformity.
\end{abstract}

A net $\left\{x_{j}\right\}_{j}$ of elements of a complete lattice $L$ order-converges to a when $\operatorname{Liminf}\left\{x_{j}\right\}=\operatorname{Lim} \sup \left\{x_{j}\right\}=a[1]$. We say that a net $\left\{f_{j}\right\}_{j}$ of functions on a set $X$ to a complete lattice $L$ uniformly order-converges to $f$ if for every subset $A$ of $X$ :

(1) $\wedge f(A) \leqslant \operatorname{Liminf}\left\{\wedge f_{j}(A)\right\}$

(2) $\bigvee f(A) \geqslant \operatorname{Lim} \sup \left\{\bigvee f_{j}(A)\right\}$

The interval topology on a given complete lattice $L$ is that defined by taking the closed intervals of $L$ as subbase for the closed sets. Any complete lattice is compact for the interval topology [2]. It is well known that usually the interval topology is not Hausdorff and the order-convergence is not topological. However any direct product of complete chains is Hausdorff for the interval topology and its convergence is the order-convergence [2]. A completely distributive complete lattice is isomorphic to a closed sublattice of a direct product of complete chains (Raney's Theorem [3, 1]). Hence for a completely distributive complete lattice $L$ the inteval topology is the uniform topology of a compact Hausdorff uniformity $\mathcal{Q}_{L}$ and its convergence is the order-convergence.

THEOREM. $A$ net $\left\{f_{j}\right\}_{j}$ of functions on a set $X$ to a completely distributive complete lattice $L$ uniformly order-converges to $f$ if and only if it uniformly converges to $f$ relatively to $\mathcal{Q}_{L}$.

Proof. A net of functions with values in a direct product of complete chains is uniformly (order-)convergent if and only if its projections in the coordinate spaces are uniformly (order-)convergent. Hence by the above quoted Raney Theorem it is enough to prove the Theorem when $L$ is a complete chain $C$.

Let $C$ be a chain with universal bounds 0,1 and $U_{C}$ its uniformity. Suppose $\left\{f_{j}\right\}$ is uniformly order-convergent to $f$ : we will show that it is uniformly convergent to $f$ relative to $\mathcal{U}_{C}$. Let $V$ be a member of $\mathcal{U}_{C}$. By the compactness of $\mathcal{U}_{C}$ there is a finite open cover $\left\{V_{i}\right\}_{i}$ of $C$ such that $V \supset \bigcup_{i}\left(V_{i} \times V_{i}\right)$. Since every finite open cover of the chain $C$ has a finite refinement consisting of closed intervals, there exists a finite family $\left\{I_{k}\right\}_{k=1}^{n}$ of closed intervals such that for every $k, I_{k} \subset V_{i(k)}$ for some $i(k)$ and

Received by the editors February 17, 1983.

1980 Mathematics Subject Classification. Primary 54H12, 06D10; Secondary 54C20, 54C30.

(C) 1984 American Mathematical Society $0002-9939 / 84 \$ 1.00+\$ .25$ per page 
$\cup_{k=1}^{n} I_{k}=C$. Setting $A_{k}=\left\{x \in X: f(x) \in I_{k}\right\}$, we have $\left[\wedge f\left(A_{k}\right), \vee f\left(A_{k}\right)\right] \subset V_{i(k)}$ for $1 \leqslant k \leqslant n$. Since $V_{i(k)}$ is open, by the uniform order-convergence of $\left\{f_{j}\right\}$ to $f$ there exists $j_{k}$ such that for every $j \geqslant j_{k}$, [ $\left.\wedge f_{j}\left(A_{k}\right), \vee f_{j}\left(A_{k}\right)\right] \subset V_{i(k)}$. Choose $j_{0} \geqslant j_{k}$ for every $1 \leqslant k \leqslant n$. We obtain for every $k\left[\wedge f\left(A_{k}\right), \vee f\left(A_{k}\right)\right] \times$ $\left[\wedge f_{j}\left(A_{k}\right), \vee f_{j}\left(A_{k}\right)\right] \subset V_{i(k)} \times V_{i(k)} \subset V$. Hence by $\cup_{k=1}^{n} A_{k}=X$ the net $\left\{f_{j}\right\}$ uniformly converges to $f$.

Now suppose $\left\{f_{j}\right\}$ is uniformly convergent to $f$ with respect to uniformity $\mathcal{Q}_{C}$. We will verify property (1) (dually the proof of (2) is obtained). Let $A$ be a subset of $X$. If $[\wedge f(A), 1]$ is open, there is $j_{0}$ such that $\left\{f_{j}(x): j \geqslant j_{0}, x \in A\right\} \subset[\wedge f(A), 1]$, since the set $[0, \wedge f(A))^{2} \cup[\wedge f(A), 1]^{2}$ is a member of $\mathscr{Q}_{C}$ and the net $\left\{f_{j}\right\}$ uniformly converges to $f$. Hence property (1) follows. Otherwise if $[\wedge f(A), 1]$ is not open, $\wedge f(A)=\vee\{b \in C: b<\wedge f(A)\}$. Let $b<\wedge f(A)$. The set $[0, \wedge f(A))^{2} \cup(b, 1]^{2}$ is a member of $\mathcal{U}_{C}$. Hence by the uniform convergence of $\left\{f_{j}\right\}$ to $f$, there is $J_{0}$ such that $\left\{f_{j}(x): x \in A, j \geqslant j_{0}\right\} \subset(b, 1]$. Then $\operatorname{Liminf}\left\{\wedge f_{j}(A)\right\} \geqslant b$ for every $b<$ $\wedge f(A)$. This implies property (1).

As a simple consequence of the above Theorem, we obtain a purely ordered characterization of the notion of equicontinuity.

Let $L$ be a complete lattice and $X$ a topological space. A function $f$ on $X$ to $L$ is lower (upper) semicontinuous at $x_{0}$ if $\operatorname{Liminf}_{x \rightarrow x_{0}} f(x) \geqslant f\left(x_{0}\right)\left(\operatorname{Limsup}_{x \rightarrow x_{0}} f(x) \leqslant\right.$ $f\left(x_{0}\right)$ ). A family $\mathbf{F}$ of functions on $X$ to $L$ is lower (upper) equi-semicontinuous if for every subfamily $\mathbf{A} \subset \mathbf{F}$ the function $\wedge \mathbf{A}(\vee \mathbf{A})$ is lower (upper) semicontinuous. This definition of lower (upper) equi-semicontinuity agrees with the usual one for a closed interval of the real field completed by adjoining universal bounds $-\infty$ and $+\infty$.

COROllary. Let $L$ be a completely distributive complete lattice and $\mathscr{Q}_{L}$ its uniformity. A family $\mathbf{F}$ is equi-continuous at $x_{0}$ with respect to $\mathcal{U}_{L}$ if and only if $\mathbf{F}$ is both lower and upper equi-semicontinuous at $x_{0}$.

In view of the Raney Theorem, our Theorem entails

Generalized Dini Theorem. Let $X$ be a topological compact space and let $L$ be a completely distributive complete lattice. If a net $\left\{f_{j}\right\}_{j}$ of continuous functions on $X$ valued in $L$ increasingly pointwise converges to a continuous function $f$, then $\left\{f_{j}\right\}_{j}$ uniformly order-converges to $f$.

\section{REFERENCES}

1. G. Birkhoff, Lattice theory, 3rd ed., Amer. Math. Soc. Colloq. Publ., vol. 25, Amer. Math. Soc., Providence, R.I., 1967.

2. O. Frink, Topology in lattices, Trans. Amer. Math. Soc. 51 (1942), 569-583.

3. G. N. Raney, A subdirect-union representation for completely distributive complete lattice, Proc. Amer. Math. Soc. 4 (1953), 518-522.

Dipartimento di Matematica, Universita di Trento, 38050 Povo (TN), Italy 